\title{
Analysis on Wavelet Family based Channel Estimation for MIMO-OFDM System
}

\author{
A. Raja, V. Thulasibai
}

\begin{abstract}
Channel estimation for (MIMO-OFDM) is an important part for present and future generation broadband wireless communications. OFDM, which uses for the spaced subcarriers to improving the performance. The channel estimation schemes based on pilot reduces the transmission rate and spectral efficiency. Many conventional schemes of channel estimation are not effective in reducing noise. It leads to poor quality signals at receiver at final stage. To rectify this problem, in this paper a wavelet family based channel estimation technique, is proposed and analyzed. The proposed research aims to estimate channels and reconstruct signal via wavelet transform, dyadic wavelet transform and fractional spline wavelet transform which enhances the spectrum efficiency and transmission rate. Simulation results shows that the fractional spline wavelet transform performs well for channel estimation and data signal reconstruction.
\end{abstract}

Keywords: Channel Estimation, Dyadic Wavelet Transform, Orthogonal Frequency Division Multiplexing (OFDM), Fractional Spline Wavelet Transform, Spectral Efficiency, Wavelet Transform.

\section{INTRODUCTION}

In OFDM the signal is modulated with specific frequency and bandwidth for which it uses the spaced subcarriers. OFDM techniques [1][2][3] are generally used in Audio video and Digital television broadcast, wireless networks, Wi-Fi (standards like 802.11a, 802.11ac etc). The receiver will receives the overall signal, which can be again demodulated to retrieve original data's. The signals demodulate with Fast Fourier Transform algorithms. Multiple input multiple output OFDM is an emerging technique for broadband wireless communication. MIMO OFDM [4] is compactable with Dipole Antenna, Multiple Antenna which provides Radio Frequency Connectors to mount other antenna types. It increases RF diversity hence no need of increasing the size of the antenna. Different antenna configuration such as $2 \times 2,3 \times 3,4 \times 4,8 \times 8$ networks are can be utilized and give good data transmission at the same time with different antennas. It gives Improved Signal strength and increased throughput of data transmitted.

Revised Manuscript Received on December 11, 2019

* Correspondence Author

A. Raja, Assistant Professor (SG), Electronics and Communication Engineering Department, Saveetha School of Engineering, Saveetha Institute of Medical And Technical Sciences, (SIMATS) Chennai, Tamil Nadu, India

Dr. V. ThulasiBai, Professor, Electronics and Communication Engineering Department, KCG College of Technology, Chennai, India.

\section{RELATED WORK}

\section{A. System Model}

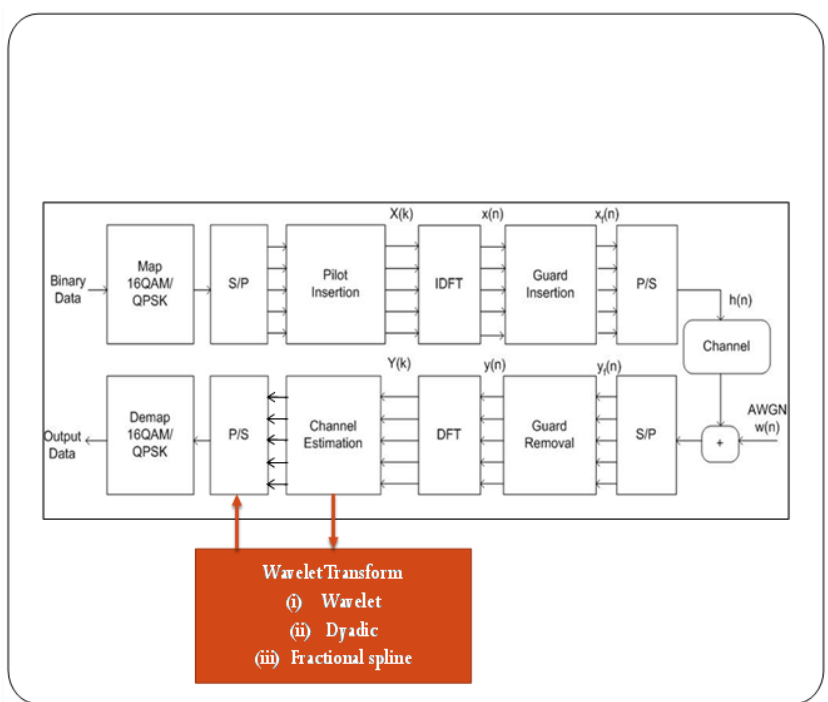

Fig.1 Block diagram for Wavelet family-based Channel Estimation

Generally, in SISO-OFDM (Single input single output system) scenario: Data goes through loss with minimum signal reception at the receiver. It requires (LOS) line of sight Antenna. Figure 1 illustrates the block diagram of MIMO-OFDM scheme for wavelet family-based channel estimation. Reduced signal strength due to RF Signal reflection. In single carrier system ISI occurs. Pilot based scheme are used for channel estimation which reduces data transmission rate. Frequency domain-based channel estimation using pilots is discussed in [5]. Conventional schemes have reduced spectral efficiency and reduced data reliability. Also provides less noise suppression. To minimize the computational difficulties Linear Minimum Mean Square Error (LMMSE) channel estimation approach is described in [8].suppression of noise threshold scheme is proposed in [9], Minimum Mean Square Error (MMSE) in [7], Least Square error (LS scheme) in [6], decision feedback based channel estimation using Haar Wavelet [10], wavelet based channel estimation for ofdm system [11] and so many schemes.

\section{B. MIMO OFDM}

The signal in MIMO network undergoes attenuation. At the receiver section the data signals in MIMO network reconstruct with wavelet transform, Dyadic wavelet

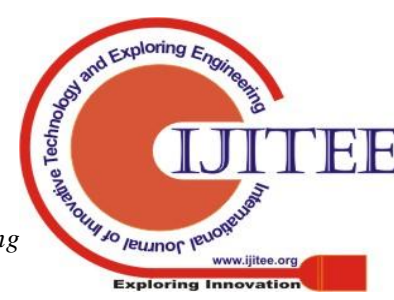


transform and Fractional spline wavelet transform [12].

The channel estimation is the process of identifying the changes occurred in the transmitted signal through the physical medium. By performing channel estimation, the receiver can achieve maximum gain in archiving transmitted data with the limited knowledge over the wireless channels. The major objectives of estimating the channel is to evaluate the several issues encountering by the wireless channel over the known and partly known set of data's that are transmitted. The subcarriers in the communication channel were placed tightly, and the method is implemented in high-speed applications where the computational estimation delay is less.[12] The wavelet family-based channel estimation methods provide higher data rate in transmission and spectrum efficiency. The wavelet denoising and decomposition reduces and suppresses the AWGN (Additive white Gaussian noise) available at the channel coefficients which are effectively estimated in time-domain. Since the frequency selectivity has powerful conditions and channels are dynamic, the proposed wavelet family provides improved channel estimation with the accuracy.

\section{PROPOSED METHOD}

Some of the channel parameters are the Doppler spread of paths related to a tap, shift in a Doppler for a path, distance between transmitter and receiver with respect to the Communication bandwidth, mobile Carrier frequency velocity, change of path amplitude with respect to time scale, change in path phase with respect to time scale, Coherence time and bandwidth, Delay spread etc. we also consider some type of channels like, slow and fast fading, , Frequency selective fading, flat fading and under spread etc. In proposed scheme, we use MIMO-OFDM (Multiple input multiple output) system scenario are to eliminate the problems in conventional scheme, we proposed wavelet family-based channel estimation. Wavelet family-based channel estimation provides Increases the Performance of MIMO (Multiple Input Multiple Output) System. Better noise suppression and efficient channel estimation, Fine Reconstruction of data from transmitted signals are achieved. Hence recently more concentration has changed towards increasing the spectral efficiency.

\section{RESULT AND DISCUSSION}

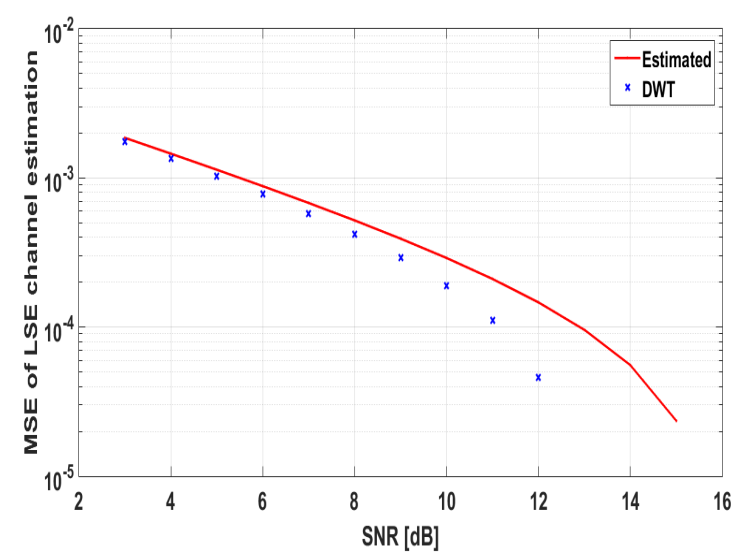

Fig 2 Channel estimation with Dyadic wavelet transform

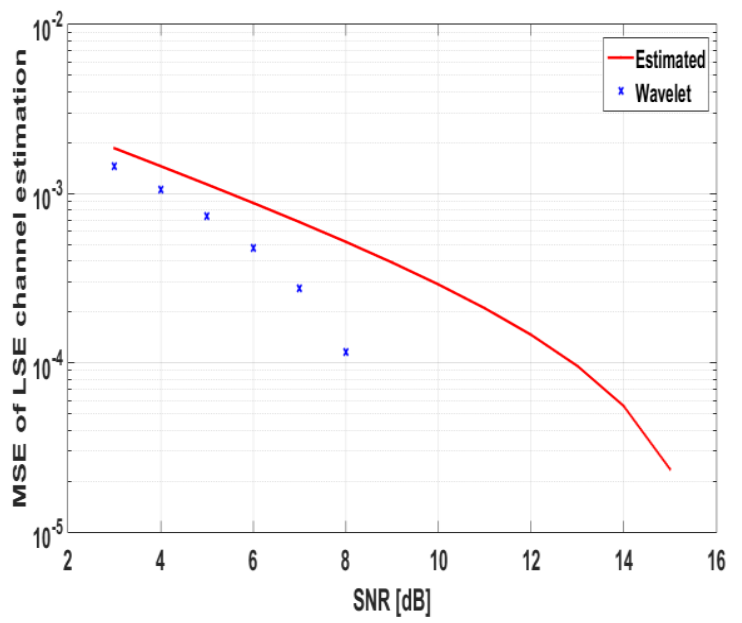

Fig 3 Channel estimation with Wavelet transform

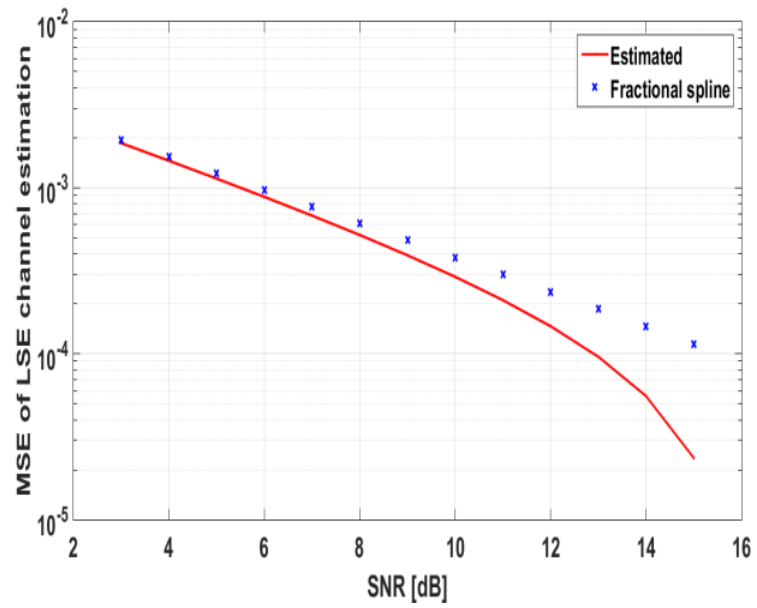

Fig 4 Channel estimation with fractional spline Wavelet transform

Most thoughtfully comparing the contrast between the performances grows nature provided by strategic communication by MIMO methods. A strategic communication scheme first provides a power gain Significant part of the power gain is notably very low with respect to signal to noise ratio range and also in the high SNR range bandwidth is limited whereas in systems it is power limited. Figure 2 shows the estimated channel. The dyadic wavelet transform applied for channel estimation and its error level. Figure 4 shows the Channel estimation with Fractional spline wavelet transforms. The wavelet transforms and fractional spline wavelet transform channel estimation technique is illustrated in figure $3 \& 4$. The Wavelet transform estimates the channel with high error rate compared to fractional spline wavelet transform. In fractional spline wavelet transform the technique overshoots the estimated level of error. So, let's say, MIMO scheme can able to offer a number of sole factors that are essential for gain and power gain. Thus, MIMO methods become the major tool to raise the capacity significant in the high SNR range.so we propose to estimate channels and reconstruct signal via wavelet transform, dyadic wavelet transform and fractional spline wavelet transform. 


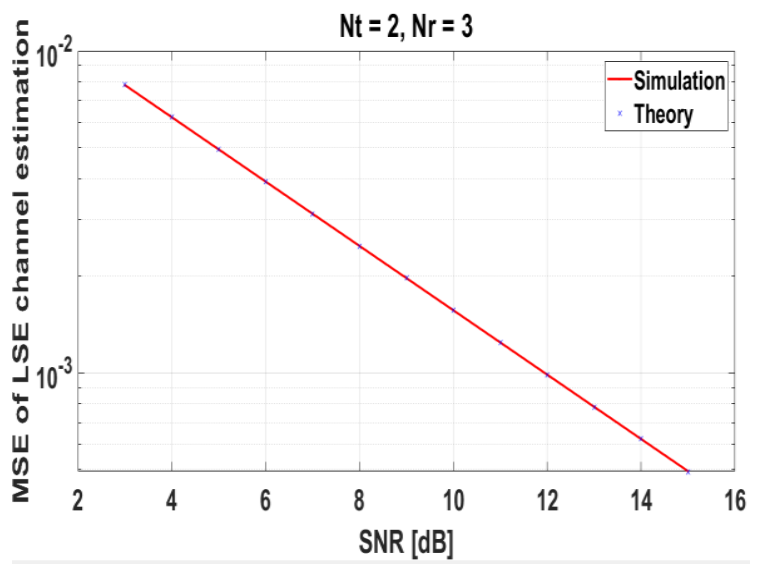

Fig 5 LS-MSE-MIMO-OFDM

The mean squared error of the least square channel calculated through the simulation results is compared with the theory. The red colour line indicates the LS channel simulations and the blue colour dot indicates the theory. Figure 5 shows the mean square error of least square error channel estimation and SNR [dB] in the MIMO-OFDM, and channel estimation level ranges from 2 to 16 in $\mathrm{SNR}[\mathrm{dB}]$ and MSE of LSE channel estimation ranges from 10-3 to 10-2. The MSE and LS simulation results initiated from 10-2 and it gradually decreases to 15 . Here number of transmit antenna $\mathrm{Nt}$ is 2 as well as Number of receive antenna $\mathrm{Nr}$ is 3 .

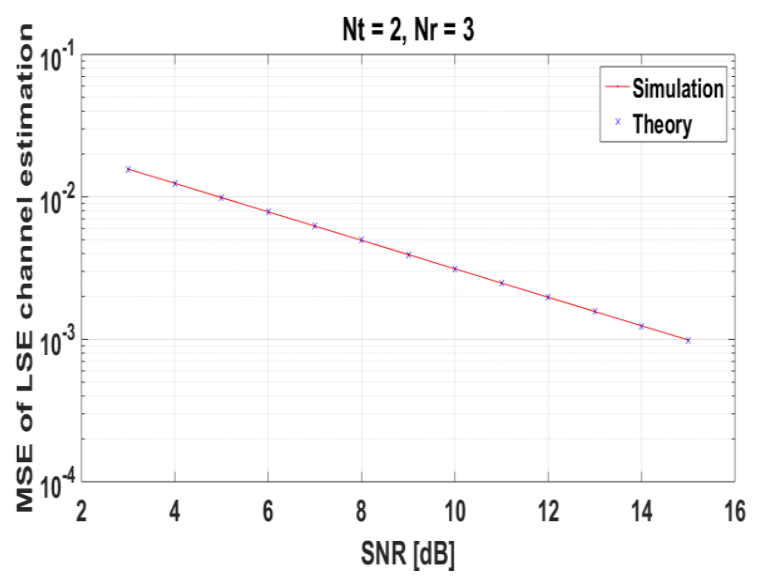

Fig 6 OFDM estimation for 64- subcarrier

Figure 6 shows the estimated output for 64-subcarriers. Mean square error of least square error channel estimation ranges from 10-4 to 10-1 and the corresponding values SNR [dB] ranges from 2 to 16 . As well as the plots form with 2 number of transmit antenna and 3 number of receiving antenna. The plots Least Square Error starts from 3 in SNR[dB] near 10-2 in MSE of LSE estimation and end at sharply 10-3 point for the estimation values and 15 th in the $\mathrm{SNR}[\mathrm{dB}]$ gradually decreased.

\section{CONCLUSION}

Furthermore, at the receiver and transmitter the number of antennas side plays a role in channel estimation and noise level present in the MIMO network. The above analysis is done for MIMO networks with two transmit antennas, three receiver antenna models. The channel in MIMO network estimates with Wavelet, Dyadic and Fractional spline wavelet transform. The data signal losses incurred during transmission and reception in MIMO network reconstruct with wavelet family algorithms. The algorithm efficiency evaluates in terms of bit error rate. The analysis shows the fractional spline wavelet transform performs well for channel estimation and data signal reconstruction. The proposed wavelet scheme needs to be analysed for different data such as image, signal, underwater acoustic communication radar communication, and optical communication.

\section{REFERENCES}

1. Liu, Y.S.; Tan, Z.H.; Hu, H.J.; Cimini, L.J.; Li, G.Y. Channel estimation for OFDM. IEEE Commun. Surv. Tutor. 2014, 16, 1891-1908.

2. X. Guo, Y.Guo, S.Li, "Experimental Investigation of Zadoff-Chu Matrix Precoding for Visible Light Communication System with OFDM Modulation", Advances in Condensed Matter Physics, 2018.

3. Y. Zhao, H.Yu, G. Wei, F.Ji, F.Chen, F. "Parameter estimation of wideband underwater acoustic multipath channels based on fractional Fourier transform", IEEE Transactions on Signal Processing,vol. 64, no (20), pp.5396-5408, 2016.

4. P.Zhu, W.Tong, J. Ma, M. Jia, M. "U.S. Patent No. 8,406,118. Washington, DC: U.S. Patent and Trademark Office, 2013.

5. Z. Liu, J.Y.Kim, D.S. Wu, D. J. Richardson, R. Slavík, "Homodyne OFDM with optical injection locking for carrier recovery. Journal of Lightwave Technology, vol. 33, no. 1, pp.34-41, 2014.

6. H.M. Son, H. Yu, J.W. Kim, J. H. Park, N.J. Lee, C. Jeong, 'U.S. Patent No. 10,181,965. Washington, DC: U.S. Patent and Trademark Office, 2019.

7. H. Ye, G.Y. Li, B.H. Juang, "Power of deep learning for channel estimation and signal detection in OFDM systems", IEEE Wireless Communications Letters, vol. 7, no. 1, pp. 114-117, 2017.

8. H. Ye, G.Y. Li, B.H. Juang, B. H" Power of deep learning for channel estimation and signal detection in OFDM systems", IEEE Wireless Communications Letters, vol. 7, no. 1, pp.114-117, 2017.

9. Y. Li, C.Tao, G. Seco-Granados, A. Mezghani, A.L. Swindlehurst, L.Liu, "Channel estimation and performance analysis of one-bit massive MIMO systems", IEEE Transactions on Signal Processing, vol.65, no. 15, pp 4075-4089, 2017.

10. Ruiguang Tang, Xiao Zhou and Chengyou Wang "A Haar Wavelet Decision Feedback Channel Estimation Method in OFDM Systems", Appl. Sci. 2018, 8, 877.

11. Mahesh Kumar Gupta, Sarika Shrivastava, A.S. Raghuvanshi and S.Tiwari "Channel estimation for wavelet based OFDM system," published in the proceedings of 2011 IEEE Int. Conf. on Devices and Communications (ICDeCom)., Publication Year: 2011, Page(s): 1 - 4

12. David Tse, University of California, Berkeley \& Pramod Viswanath, University of Illinois, Urbana-Champaign, Fundamentals of Wireless Communication, published by Cambridge University Press, which owns the copyright, September 10, 2004.

13. G. Shilpa, "Low power 128 point split radix FFT for LTE application", International Journal of MC Square Scientific Research, Vol.6, No.1 Nov 2014,pp.66-74.

\section{AUTHORS PROFILE}

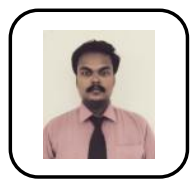

A. Raja is Assistant Professor (SG) in Electronics and Communication Engineering Department, Saveetha School of Engineering, Saveetha Institute of Medical And Technical Sciences, (SIMATS) Chennai, Tamil Nadu, India.

Dr. V. ThulasiBai is Professor in Electronics and Communication Engineering Department, KCG College of Technology, Chennai, India. 